\title{
Spherical approximating and interpolating moving least squares in geodesy and geophysics: a case study for deriving gravity acceleration at sea surface in the Persian Gulf
}

DOI: https://doi.org/10.1515/jogs-2020-0112

Received November 2, 2019; accepted May 25, 2020

\begin{abstract}
This paper is aimed at introducing the concept of Spherical Interpolating Moving Least Squares to the problems in geodesy and geophysics. Based on two previously known methods, namely Spherical Moving Least Squares and Interpolating Moving Least Squares, a simple theory is formulated for using Spherical Moving Least Squares as an interpolant. As an application, a case study is presented in which gravity accelerations at sea surface in the Persian Gulf are derived, using both the approximation and interpolation mode of the Spherical Moving Least Squares. The roles of the various elements in the methods-weight function, scaling parameter, and the degree of spherical harmonics as the basis functions-are investigated. Then, the results of approximation and interpolation are compared with the field data at sea surface, collected by shipborne gravimetry approach. Finally, the results are compared with another independent interpolation method-spline interpolation. It is shown that in this particular problem, SMLS approximation and SIMLS interpolation present a better accuracy than spherical splines.
\end{abstract}

Keywords: gravity acceleration values at sea surface, scaling parameter, shipborne gravimetry, spherical harmonic basis functions, spherical interpolating moving least squares, spherical moving least squares, weight function

\section{Introduction}

With the technological advances in geodesy and geophysics, there has always been the need to devise new mathematical methods.

\footnotetext{
*Corresponding Author: M. Kiani: School of Surveying and Geospatial Data Engineering, University of Tehran, Tehran, Iran., E-mail: mostafakiani@ut.ac.ir
}

The interpolation and approximation problems that frequently appear in geodesy and geophysics are of key importance for geodetic community and as the result, geodesists and geophysicists have investigated many different approximants and interpolants. The observations in geodesy and geophysics are almost never continuously sampled, i.e. they are not known in every point and do not have an analytical formula. Therefore, the behavior of the function under consideration is not known. Some assumptions may be made to resolve this problem. One of the most widely used assumptions is smoothness. This is because provably most of the functions in geodesy and geophysics are smooth, especially when one considers the gravity field functions-potential and acceleration. This assumption leads to the concept of spline interpolation and approximation. There have been many works on the theoretical and applied aspects of the spline functions, including (Freeden, et al, 2018a), (Freeden, et al, 1998), (Freeden, 2009), (Freeden, 1981), (Kiani , 2019b), (Kiani , 2020), (Wahba, 1981), and (Wahba, 1990).

Since the observations in geodesy and geophysics are commonly either on the surface of the Earth or outside of the Earth's surface in 3D space, two different approaches have been introduced. First is the surface spherical spline (see many contributions such as (Freeden, 1981) and (Wahba, 1981).) In this method, it is assumed that the data are scattered on the surface of the sphere. The Green's functions play a paramount role in the definition and implementation of these surface spline functions. Second is the outer, three-dimensional spline, defined based on integral kernels such as Abel-Poisson. A number of different works have been devoted to the spherical and ellipsoidal case of the spline functions, including (Akhtar, et al., 2012), (Freeden, et al, 2018a), (Freeden, et al, 1998), and (Freeden, 2009). Spline functions have powerful characteristics that make them one of the most ideal classes of interpolants and approximants. These include smoothness, differentiability, and minimality of (semi-)norm in the context of a Hilbert space. Based on these character- 
istics many papers have used thsese functions to interpolate data, including (Baramidze, et al., 2006). However, these functions have some drawbacks that make their implementation unfavorable, hard, or even impossible. One of the most important drawbacks of the spline functions is their computational complexity: if $J$ points are to be interpolated, solving a $J \times J$ system of normal equations is required. This is because like the correlation functions the kernel based on which the spline function is defined must be computed for every two point-pair, even when the two points are the same. One can understand the huge system of normal equations that must be solved for the usually large number of interpolation points. Although the modern computers can handle such large number of equations, we are interested in finding more efficient ways of interpolation of data.

Other methods of approximation and interpolation, such as Radial Basis Functions (RBFs) and hyperinterpolation, have also other problems: the Lebesgue constants in the projection process to spherical harmonics are not all bounded in a uniform manner (see references such as (Wendland, 2000).) Hence, a method of approximating and interpolating data seemed necessary and many researchers investigated a new class of approximants called Moving Least Squares (MLS), starting by (Lancaster, et al, 1981). There are many advantages to MLS approximants, including local approximation (by having a locally supported weight function), the small system of equations that must be solved for each point, and the ability to be used for both the regular grids and meshless points. As for the need of Geosciences, Spherical Moving Least Squares (SMLS) was devised by (Wendland, 2000). SMLS is used when the data to be approximated are on the surface of the sphere, thus rendering it quite a powerful tool to approximate Geodetic data, most importantly gravity data in Earth's gravity field.

MLS can be used as an interpolant and in that case it is usually called IMLS or Interpolating Moving Least Squares (refer to (Dawes, et al, 2007),(Kawano, et al, 2006), and (Maisuradze, et al, 2003) for more details.) One instance of the Geodetic applications of MLS method as an interpolant is introduced in (Kiani , 2019a) in which the MLS method has been used to locally interpolate geoid height. Since in many cases in geodesy and geophysics it is essential to assume that the data are on the surface of the Earth, which is usually considered a sphere, the present paper is aimed at developing the concept of Spherical Interpolating Moving Least Squares (SIMLS.) The application presented in this paper-producing gravity acceleration values at sea surface in the Persian Gulf-is implemented using the results obtained for SIMLS. The mentioned advantages of MLS-local approximation property, less computational complexity, and the meshless approximation property-are all true of SMLS and SIMLS, as well.

The rest of this paper is organized as follows. In section 2, the mathematical background of the SMLS method is presented and the SIMLS concept is developed and defined. In section 3, the SMLS approximant and the SIMLS interpolant are used to derive the gravity acceleration at sea surface in the Persian Gulf. The discussions about the results is also presented in this section. Finally, the conclusions are stated in section 5 .

\section{Derivation of SIMLS}

In this section the definition of the SIMLS interpolant is presented and mathematical formulae for its applications are derived. In order to define the concept of SIMLS, we need to overview the concept of SMLS. In what follows, an overview of the SMLS method is presented and based on that the SIMLS method is developed.

\subsection{Overview of the SMLS}

The point of departure for the definition of SIMLS interpolant is SMLS approximant. According to (Wendland, 2000), MLS approximant, $P_{S M L S}$, for the continuous function $F$, defined on the two-dimensional sphere $\Omega$, is the solution of the following minimization problem

$$
\left\|F-P_{S M L S}\right\|_{W} \rightarrow \min ,
$$

where the minimization is performed at known points $\xi_{i}, i=1, \ldots, M$, and $W$ is the weight matrix. This matrix, constructed by a weight function $\omega: \Omega \times \Omega \rightarrow[0, \infty]$, is usually written in the following form

$$
W=\operatorname{diag}\left[\omega\left(\eta, \xi_{1}\right), \ldots, \omega\left(\eta, \xi_{M}\right)\right],
$$

where $\eta$ is the point at which the approximate value is to be found. In this paper we use two different weight functions, namely Gaussian and Spline Weight Function (SWF.) These weight functions are defined, accordingly, as (see (Cheng, et al., 2008) and references therein)

$$
\begin{gathered}
\omega_{G}\left(\eta, \xi_{i}\right)=\frac{e^{-\left(\epsilon d\left(\eta, \xi_{i}\right)\right)^{2}}-e^{-\epsilon^{2}}}{1-e^{-\epsilon^{2}}} \\
\omega_{S W F}\left(\eta, \xi_{i}\right)=1-6 d^{2}\left(\eta, \xi_{i}\right)+8 d^{3}\left(\eta, \xi_{i}\right)-3 d^{4}\left(\eta, \xi_{i}\right),
\end{gathered}
$$

where $0<\epsilon<1$ is a small, positive value that can be set arbitrarily, or be determined by a certain criterion in each 
particular problem, and $d\left(\eta, \xi_{i}\right)$ is what we call "normalized distance", defined as the following based on the scaling parameter $\delta$

$$
d\left(\eta, \xi_{i}\right)=\frac{\left\|\eta-\xi_{i}\right\|}{\delta}, \quad 0 \leq d \leq 1 .
$$

The role of the parameter $\delta$ is to determine the neighbor points, i.e. points that lie within a local vicinity. Thus, the set of neighbor points to the approximation point $\eta$ is defined as

$$
N_{e}(\eta)=\left\{\xi_{i} \mid\left\|\eta-\xi_{i}\right\| \leq \delta\right\} .
$$

The explicit Least Squares (LS) solution of Eqn.(1) is a linear composition of the spherical harmonics ((Wendland, 2000).) It is important to notice that for solving the minimization problem in Eqn.(1) there must be chosen a set of basis functions. By choosing the spherical harmonics up to degree $q, H_{j, k-1}, j=0, \ldots, q, \quad k=1, \ldots, 2 j+1$, as the basis functions, we arrive at the $q^{\text {th }}$ degree SMLS approximation. Hence, we have the following linear composition

$$
P_{S M L S}(\eta)=\sum_{i=1}^{M} b_{i}(\eta) F\left(\xi_{i}\right)
$$

where

$$
b_{i}(\eta)=\omega_{\ell}\left(d\left(\eta, \xi_{i}\right)\right) \sum_{j=0}^{q} \sum_{k=1}^{2 j+1} c_{j, k} H_{j, k-1}\left(\xi_{i}\right), \ell=G, S W F,
$$

in which $c_{j, k}, j=0, \ldots, q, k=1, \ldots, 2 j+1$ are the unique solution of the following system of equations

$$
\begin{aligned}
& \sum_{j=0}^{q} \sum_{k=1}^{2 j+1} \sum_{i=1}^{M} c_{j, k} \omega_{\ell}\left(d\left(\eta, \xi_{i}\right)\right) H_{j, k-1}\left(\xi_{i}\right) H_{j^{\prime}, k^{\prime}-1}\left(\xi_{i}\right) \\
& =H_{j^{\prime}, k^{\prime}-1}(\eta), \\
& j^{\prime}=0, \ldots, q, k^{\prime}=1, \ldots, 2 j^{\prime}+1, \quad \ell=G, S W F .
\end{aligned}
$$

It is important to notice that the basis functions $H$ are spherical harmonics, defined based on the Legendre and cosine and sine functions as the following in the spherical co-latitude $(\theta)$ and longitude $(\lambda)$ coordinates

$$
\begin{aligned}
& H_{j, k}\left(\xi_{i}\right)=P_{j, k}\left(\cos \theta_{\xi_{i}}\right) \cos \left(k \lambda_{\xi_{i}}\right), \quad j=0, \ldots, q, \\
& k=0, \ldots, j, \\
& H_{j, k+q}\left(\xi_{i}\right)=P_{j, k}\left(\cos \theta_{\xi_{i}}\right) \sin \left(k \lambda_{\xi_{i}}\right), \quad j=0, \ldots, q, \\
& k=1, \ldots, j .
\end{aligned}
$$

For instance, the following are the basis functions used for approximation and interpolation when the harmonic basis functions are used up to degree $q=2$

$$
\begin{aligned}
& H_{0,0}\left(\xi_{i}\right)=1, \\
& H_{1,0}\left(\xi_{i}\right)=P_{1}\left(\cos \theta_{\xi_{i}}\right), \\
& H_{1,1}\left(\xi_{i}\right)=P_{1,1}\left(\cos \theta_{\xi_{i}}\right) \cos \left(\lambda_{\xi_{i}}\right), \\
& H_{1,2}\left(\xi_{i}\right)=P_{1,1}\left(\cos \theta_{\xi_{i}}\right) \sin \left(\lambda_{\xi_{i}}\right), \\
& H_{2,0}\left(\xi_{i}\right)=P_{2}\left(\cos \theta_{\xi_{i}}\right), \\
& H_{2,1}\left(\xi_{i}\right)=P_{2,1}\left(\cos \theta_{\xi_{i}}\right) \cos \left(\lambda_{\xi_{i}}\right), \\
& H_{2,2}\left(\xi_{i}\right)=P_{2,2}\left(\cos \theta_{\xi_{i}}\right) \cos \left(2 \lambda_{\xi_{i}}\right), \\
& H_{2,3}\left(\xi_{i}\right)=P_{2,1}\left(\cos \theta_{\xi_{i}}\right) \sin \left(\lambda_{\xi_{i}}\right), \\
& H_{2,4}\left(\xi_{i}\right)=P_{2,2}\left(\cos \theta_{\xi_{i}}\right) \cos \left(2 \lambda_{\xi_{i}}\right),
\end{aligned}
$$

Remark 2.1. The number of unknown coefficients to be estimated in Eqn.(8) is $(q+1)^{2}$. Thus, it is important to choose $\delta$ such that all unknowns be estimable. In fact, the scaling parameter will be considered a function of the degree of the spherical harmonics so as to fulfil this requirement.

\subsection{The derivatives of the SMLS}

The definition of the SMLS approximant in Eqn.(7) introduces the possibility of deriving some new functions, which is done by applying continuous operators. This helps to use the SMLS approximant as an analytical form based on which different values can be derived from a function. For example, having potentials, we can calculate accelerations by differentiating the SMLS approximant. Let us assume an operator, denoted here by $L$, acts on both sides of Eqn.(7)

$$
L_{\eta} P_{S M L S}(\eta)=\sum_{i=1}^{M} L_{\eta} b_{i}(\eta) F\left(\xi_{i}\right) .
$$

Considering the relation in Eqn.(8), it can be simply shown that

$$
\begin{aligned}
& L_{\eta} b_{i}(\eta)=L_{\eta} \omega_{\ell}\left(d\left(\eta, \xi_{i}\right)\right) \sum_{j=0}^{q} \sum_{k=1}^{2 j+1} c_{j, k} H_{j, k}\left(\xi_{i}\right), \\
& \ell=G, S W F .
\end{aligned}
$$

The used weight functions in this paper-Gaussian, given in Eqn.(3), and SWF, given in Eqn.(4)-are infinitely often differentiable. Thus, any continuous operator can act on them.

One particularly important operator is spherical gradient for a sphere with radius $R$. In this case we have $(\eta=$ 
$(\theta, \lambda)$, where $\theta$ is co-latitude, and $\lambda$ is longitude)

$$
\begin{aligned}
& \nabla_{\eta} b_{i}(\eta)=\sum_{j=0}^{q} \sum_{k=1}^{2 j+1} c_{j, k} H_{j, k}\left(\xi_{i}\right) \times \\
&\left(\frac{1}{R \sin \theta} \frac{\partial \omega_{\ell}\left(d\left(\eta, \xi_{i}\right)\right)}{\partial \lambda}, \frac{1}{R} \frac{\partial \omega_{\ell}\left(d\left(\eta, \xi_{i}\right)\right)}{\partial \theta}\right), \ell=G, S W F
\end{aligned}
$$

The normalized distance between the approximation point $\eta=(\theta, \lambda)$ and the scattered point $\xi_{i}=\left(\theta^{\prime}, \lambda^{\prime}\right)$ in the spherical coordinates is

$$
d\left(\eta, \xi_{i}\right)=\frac{1}{\delta} \sqrt{(x-y)^{T}(x-y)},
$$

where

$$
\begin{aligned}
& x=(R \sin \theta \cos \lambda, R \sin \theta \sin \lambda, R \cos \theta), \\
& y=\left(R \sin \theta^{\prime} \cos \lambda^{\prime}, R \sin \theta^{\prime} \sin \lambda^{\prime}, R \cos \theta^{\prime}\right) .
\end{aligned}
$$

Therefore, one can simply show that

$$
d\left(\eta, \zeta_{i}\right)=\frac{R}{\delta} \sqrt{2} \sqrt{1-\cos \theta \cos \theta^{\prime}-\sin \theta \sin \theta^{\prime} \cos \left(\lambda-\lambda^{\prime}\right)} .
$$

Using Eqn.(17) in Eqn.(3) and Eqn.(4), the relation in Eqn.(14) takes the following forms

- For $\omega_{G}\left(\eta, \xi_{i}\right)$

$$
\nabla_{\eta} b_{i}(\eta)=\sum_{j=0}^{q} \sum_{k=1}^{2 j+1} c_{j, k} H_{j, k}\left(\xi_{i}\right) \frac{-2 \epsilon^{2} R e^{-\left(\epsilon d\left(\eta, \xi_{i}\right)\right)^{2}}}{\delta^{2}\left(1-e^{\left.-\epsilon^{2}\right)}\right.} \times
$$

$\left(\sin \theta^{\prime} \sin \left(\lambda-\lambda^{\prime}\right), \sin \theta \cos \theta^{\prime}-\cos \theta \sin \theta^{\prime} \cos \left(\lambda-\lambda^{\prime}\right)\right)$.

- $\operatorname{For} \omega_{S W F}\left(\eta, \xi_{i}\right)$

$$
\nabla_{\eta} b_{i}(\eta)=\sum_{j=0}^{q} \sum_{k=1}^{2 j+1} c_{j, k} H_{j, k}\left(\xi_{i}\right)\left(-12 \frac{R}{\delta^{2}}\left(d\left(\eta, \xi_{i}\right)-1\right)^{2}\right) \times
$$

$\left(\sin \theta^{\prime} \sin \left(\lambda-\lambda^{\prime}\right), \sin \theta \cos \theta^{\prime}-\cos \theta \sin \theta^{\prime} \cos \left(\lambda-\lambda^{\prime}\right)\right)$.

The derivatives in Eqn.(18) and Eqn.(19) are important in the application we will present in section 3.

\subsection{The definition of SIMLS}

So far, an overview of the method of SMLS is presented. As stated in section 2.1, SMLS is primarily an approximant. This means that the number of points in the neighborhood of the interpolation point is greater than the number of coefficients to be estimated in Eqn.(8). It is, however, possible to use the SMLS as an interpolant, as is the case with MLS and IMLS. The number of neighbor points in the interpolation problem is determined by the degree of spherical harmonics that are used as the basis functions. Hence, one needs to explicitly determine the number of points, $M$, in Eqn.(7) to use SMLS as an interpolant. As stated earlier (Cf. Remark 2.1), there are $(q+1)^{2}$ unknown coefficients in Eqn.(8). Thus we have

$$
M=(q+1)^{2} .
$$

This means we should find the first $(q+1)^{2}$ points in the neighborhood of the interpolation point.

Observation 2.1. According to Eqn.(18) and Eqn.(19), it is essential to set the minimum of $\delta$ when one deals with the derivatives of the SIMLS. Therefore, $\delta$ is set as the minimum value of the scaling parameters such that in the neighborhood of each of the $n^{\prime}$ interpolation points $\left(\delta_{\eta_{i}}, i=1, \ldots, n^{\prime}\right)$ there are exactly $(q+1)^{2}$ points

$$
\delta=\min \left\{\delta_{\eta_{i}} \mid i=1, \ldots, n^{\prime}\right\} .
$$

It is obvious that if more than $(q+1)^{2}$ points are found in the neighborhood of interpolation points, the first $(q+1)^{2}$ points whose normalized distances to the interpolation point are smallest (in the ascending order) will be chosen.

\section{SMLS and SIMLS for deriving gravity acceleration values at sea surface}

In this section, an application of the SMLS and SIMLS is presented for deriving the gravity acceleration values at sea surface. The region of case study is the Persian Gulf, for which the method of spherical splines is presented in (Safari, et al, 2014). Note that the representation of the data in each step will be similar to those in (Safari, et al, 2014). Thus, the reader should refer to the mentioned paper to see the figures. In the present paper, the results of comparison between real data-gathered by shipborne gravimetryand computed data-derived from SMLS and SIMLS-is presented.

In this section, first we use the SMLS to approximate gravity acceleration values. Then, our new method of SIMLS will be tested. The results of the SMLS and SIMLS methods will be compared with the spherical spline interpolation in the discussion section. In what follows the 
symbol $\xi$ is used for grid points. Also, the symbol $\eta$ refers to the interpolation or approximation points.

There are 10 steps involved in using both SMLS and SIMLS for deriving the gravity acceleration values at sea surface. The following diagram summarizes these steps. The details of each step are mentioned after the diagram.

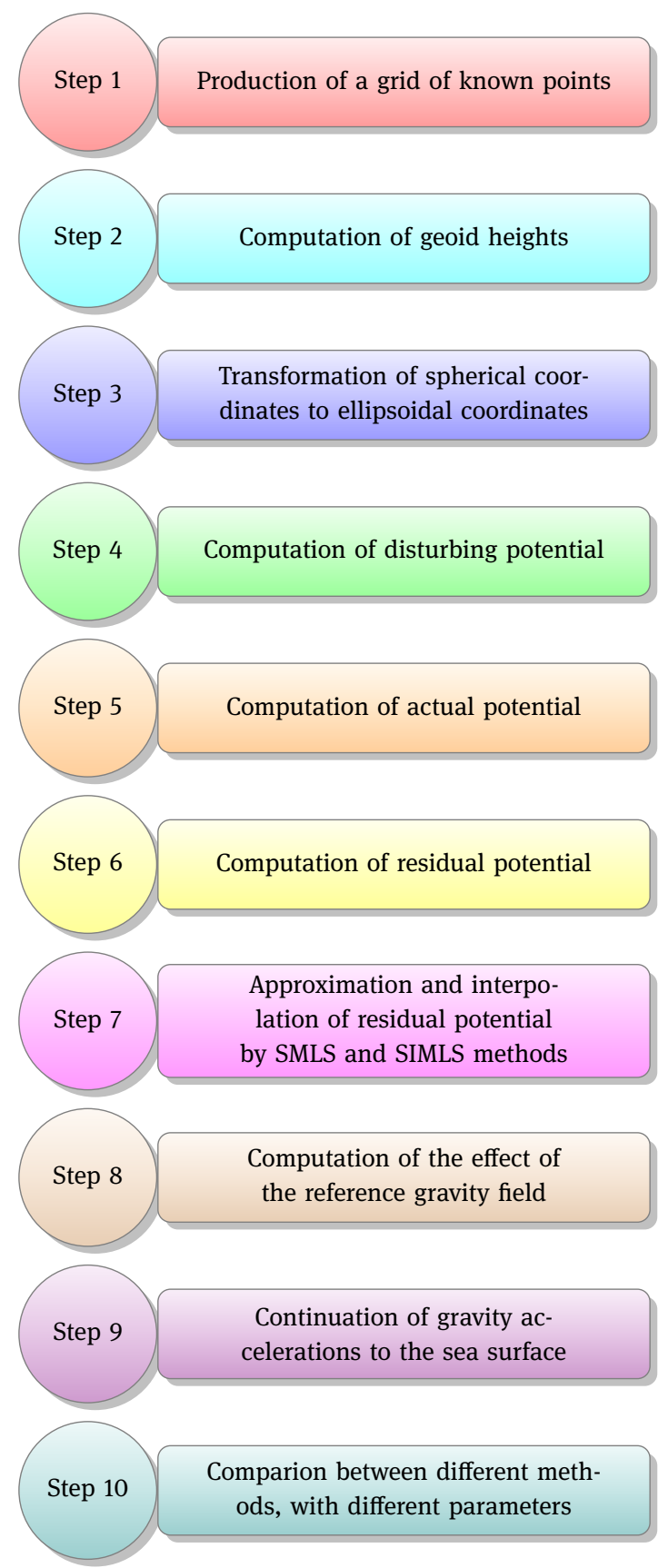

1- Producing a (semi-)regular grid of points $\left(\xi_{i}, i=\right.$ $1, \ldots, n)$ in the region. The goal in this step is to produce the scattered points based on which the gravity acceleration values will be approximated-in the case of SMLS-or interpolated-in the case of SIMLS. The region is not perfectly rectangular; therefore, the grid will be semi-regular, with some irregularities interspersed among the regular points. Roughly, the region can be described-in terms of spherical latitude and longitude-as

$$
\begin{aligned}
& 48.1 \leq \lambda \leq 64 \\
& 23.5 \leq \phi_{s} \leq 30.4
\end{aligned}
$$

The total number of points in this grid is $n=3439$. Figure 1 shows these points.

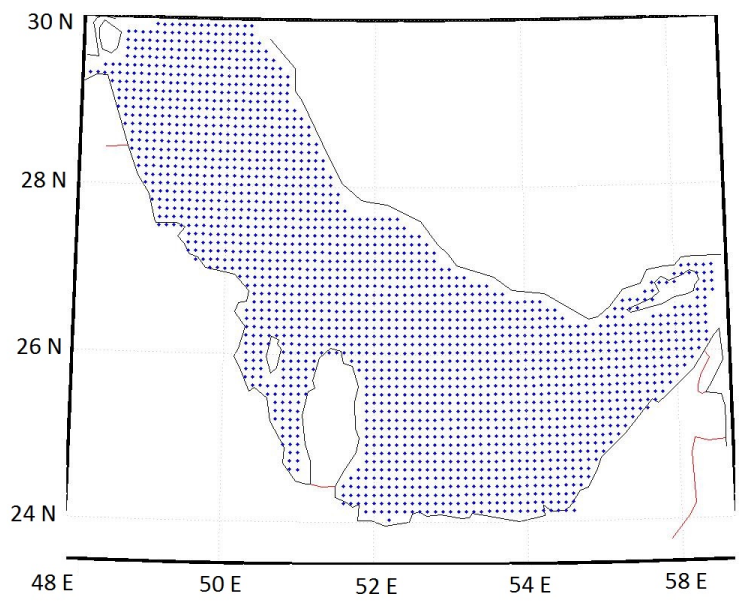

Fig. 1. Distribution of points used for computations

2- Computing geoid height from Mean Sea Level (MSL) data and Sea Surface Topography (SST, which is also known as Dynamic Topography(DT)) data. To this end, we use the MSL data from CSRMSS95 MSL model ((Kim, et al, 1995)), and SST data from the POCM4B model((Forste, et al, 1998)), which is based on the spherical harmonic expansion of the DT (up to degree and order 360), using the specified coefficients. geoid height $(N)$ is determined by adding the MSL $\left(h_{M S L}\right)$ and the SST values $\left(h_{S S T}\right)$, as the following

$$
N\left(\xi_{i}\right)=h_{M S L}\left(\xi_{i}\right)+h_{S S T}\left(\xi_{i}\right) .
$$

3- Transforming the spherical coordinates in step 1 to the ellipsoidal coordinates. The goal of this step is to provide the ellipsoidal coordinates (Gaussian system) to use in the next steps, in which the ellipsoidal formulae are used to compute the potential and the reference part of the gravity acceleration. To do this transformation, it is important to notice that the only change that will occur is in the latitude $\left(\phi_{s}^{\prime} \rightarrow \phi_{e}^{\prime}\right)$ : longitude is the same in both the spherical and ellipsoidal coordinates $\left(\lambda_{e}^{\prime}=\lambda_{s}^{\prime}=\lambda^{\prime}\right)$. Hence, using $a, e^{2}$ 
(respectively semi-major axis and eccentricity of the reference ellipsoid) and taking the relations in Eqn.(16) into account, we have $\left(\cos \theta^{\prime}=\sin \phi^{\prime}\right)$

$$
\begin{aligned}
& R \cos \phi_{s}^{\prime} \cos \lambda^{\prime}=\frac{a}{\sqrt{1-e^{2} \sin ^{2} \phi_{e}^{\prime}}} \cos \phi_{e}^{\prime} \cos \lambda^{\prime}, \\
& R \cos \phi_{s}^{\prime} \sin \lambda^{\prime}=\frac{a}{\sqrt{1-e^{2} \sin ^{2} \phi_{e}^{\prime}}} \cos \phi_{e}^{\prime} \sin \lambda^{\prime}, \\
& R \sin \phi_{s}^{\prime}=\frac{a\left(1-e^{2}\right)}{\sqrt{1-e^{2} \sin ^{2} \phi_{e}^{\prime}}} \sin \phi_{e}^{\prime} .
\end{aligned}
$$

So, one can simply show that

$$
\phi_{e}^{\prime}=\arctan \left(\frac{\tan \phi_{s}^{\prime}}{1-e^{2}}\right) .
$$

4- Computing the so-called Disturbing Potential (DP). The geoid height can be converted to the DP using the ellipsoidal Bruns formula (see (Ardalan, et al., 2001) and references therein,) using the following relation

$$
\begin{aligned}
& \delta w\left(\xi_{i}\right)=N\left(\xi_{i}\right) \times \\
& \left(\frac{\frac{G M}{b^{2}+E^{2}}\left(3 \cos ^{2} \phi_{e}^{\prime}+1\right) \frac{6 b\left(b^{2}+E^{2}\right) \cot ^{-1}\left(\frac{b}{e}\right)-3 b E+E^{2}-3 E}{6 b\left(b^{2}+E^{2}\right) \cot ^{-1}\left(\frac{b}{E}\right)-3 b E}}{\sqrt{\frac{b^{2}+E^{2} \cos ^{2} \phi_{e}^{\prime}}{b^{2}+E^{2}}}}+\right. \\
& \left.\frac{\omega^{\prime 2} b \sin \phi_{e}^{\prime}}{\sqrt{\frac{b^{2}+E^{2} \cos ^{2} \phi_{e}^{\prime}}{b^{2}+E^{2}}}}\right) \\
& i=1, \ldots, n,
\end{aligned}
$$

where $G M=3.9860 \times 10^{14} \frac{\mathrm{m}^{3}}{\mathrm{~s}^{2}}$ is the gravitational constant, $b$ the semi-minor axis of the reference ellipsoid, $E^{2}=a^{2}-$ $b^{2}$ the linear eccentricity, and $\omega^{\prime}=7.292115 \times 10^{-5} \frac{\mathrm{rad}}{\mathrm{s}}$ the angular velocity of the Earth's rotation.

Remark 3.1. One can see that the ellipsoidal coordinates, computed in the previous step, are used in the current step.

5- Computing the actual potential. This is done by adding the DP values $(\delta w)$ to the geoid potential $\left(W_{0}=\right.$ $62636855.8 \frac{m^{2}}{s^{2}}$ ((Safari, et al, 2014))), as the following

$$
W_{\text {real }}\left(\xi_{i}\right)=W_{0}+\delta w\left(\xi_{i}\right), \quad i=1, \ldots, n .
$$

6- Computing the so-called residual potential. The goal of this step is to obtain the values-of the potential type-based on which the SMLS and SIMLS approximation and interpolation will be performed. One reason to do this step is to obtain smaller values that can be approximated or interpolated better. Another reason is to have an analytical framework to perform approximation or interpolation, i.e. the most important part of the gravity acceleration-reference field effect-is determined by the analytical formula for the ellipsoidal gravity acceleration for the reference field.

To derive the residual potential values we have

$$
d W\left(\xi_{i}\right)=W_{\text {real }}\left(\xi_{i}\right)-W_{\text {ref }}\left(\xi_{i}\right) \quad i=1, \ldots, n,
$$

where the $W_{r e f}$ is computed using the ellipsoidal potential formula up to degree and order 360 , for which the spherical coefficients in the EGM96 geopotential model are transformed into the ellipsoidal ones, using the relations in (Jekeli, 1988).

Remark 3.2. Note that the $W_{\text {ref }}$ contains both the gravitational $\left(W_{g}\right)$ and the centrifugal $\left(W_{c}\right)$ compontents. In other words, one can observe that

$$
W_{r e f}\left(\xi_{i}\right)=W_{g}\left(\xi_{i}\right)+W_{c}\left(\xi_{i}\right) \quad i=1, \ldots, n .
$$

7- Using the SMLS and SIMLS methods to derive the residual gravity. In this step the methods of SMLS and SIMLS are used to produce the residual gravity acceleration values at the reference ellipsoid, at approximation or interpolation points. The approximation (or interpolation) points used in this step are taken from the shipborne gravimetry data by International Gravimetric Bureau (which is widely known as BGI institute), which works in the field of collecting gravity data at sea surface. These data contain the coordinates of the approximation (or interpolation) points-in latitude and longitude-and the values of gravity acceleration at these points. The state of the collection of data for each of the observations is included in the data, to use only those that are labeled as correct observations. The number of approximation (or interpolation) points in this step is $n^{\prime}=6657$.

According to Eqn.(12), Eqn.(18), and Eqn.(19) if the values to be approximated or interpolated are of the potential type the resulting differentiation would be of the acceleration type. Therefore, combining Eqn.(12), Eqn.(18), Eqn.(19), and Eqn.(28) one gets the following relations

- For $\omega_{G}\left(\eta, \xi_{i}\right)$

$$
\begin{aligned}
& d g_{G}\left(\eta_{i}\right)=\| \sum_{r=1}^{M} \sum_{j=0}^{q} \sum_{k=1}^{2 j+1} c_{j, k} H_{j, k}\left(\xi_{r}\right) F\left(\xi_{r}\right) \times \\
& \frac{-2 \epsilon^{2} R e^{-\left(\epsilon d\left(\eta_{i}, \xi_{r}\right)\right)^{2}}}{\delta^{2}\left(1-e^{\left.-\epsilon^{2}\right)} \times\right.} \\
& \left(\sin \theta^{\prime} \sin \left(\lambda-\lambda^{\prime}\right), \sin \theta \cos \theta^{\prime}-\cos \theta \sin \theta^{\prime} \cos \left(\lambda-\lambda^{\prime}\right)\right) \|, \\
& i=1, \ldots, n^{\prime},
\end{aligned}
$$


- For $\omega_{S W F}\left(\eta, \xi_{i}\right)$

$$
\begin{aligned}
& d g_{S W F}\left(\eta_{i}\right)=\| \sum_{r=1}^{M} \sum_{j=0}^{q} \sum_{k=1}^{2 j+1} c_{j, k} H_{j, k}\left(\xi_{r}\right) \times F\left(\xi_{r}\right) \times \\
& \left(-12 \frac{R}{\delta^{2}}\left(d\left(\eta_{i}, \xi_{r}\right)-1\right)^{2}\right) \times \\
& \left(\sin \theta^{\prime} \sin \left(\lambda-\lambda^{\prime}\right), \sin \theta \cos \theta^{\prime}-\cos \theta \sin \theta^{\prime} \cos \left(\lambda-\lambda^{\prime}\right)\right) \|, \\
& \quad i=1, \ldots, n^{\prime},
\end{aligned}
$$

in which the $\|$.$\| denotes the L_{2}$ norm.

8- Adding the effect of the reference gravity field. The removed effect of the reference field in step 6 is computed in terms of gravity acceleration. Then these values are added to the residual gravity values in the previous step. It is essential to notice that the same degree of ellipsoidal harmonic expansion (up to 360) will be used. By adding the mentioned values, we arrive at the actual gravity acceleration

$g_{\text {actual }}^{e}\left(\eta_{i}\right)=g_{\text {ref }}\left(\eta_{i}\right)+d g_{\ell}\left(\eta_{i}\right), \quad \ell=G, S W F, i=1, \ldots, n^{\prime}$,

in which $e$ denotes that the values are on the surface of the ellipsoid.

9- Adding the effect of continuation to the sea surface. In this step, we add the free-air correction to the computed values in step 8 , using the following relation

$$
g_{\text {actual }}^{\text {sea }}\left(\eta_{i}\right)=g_{\text {actual }}^{e}\left(\eta_{i}\right)-0.3086 N\left(\eta_{i}\right) .
$$

Note that the free-air correction in this region is positive since the geoid height is negative.

Remark 3.3. Notice that $N\left(\eta_{i}\right), i=1, \ldots, n^{\prime}$ are different from $N$ in Eqn.(23). Thus, based on $N\left(\xi_{r}\right), r=1, \ldots, n$ the values $N\left(\eta_{i}\right), i=1, \ldots, n^{\prime}$ are interpolated or approximated, using the same approximant or interpolant used for computing $g_{\text {actual }}^{e}\left(\eta_{i}\right), i=1, \ldots, n^{\prime}$.

10-Comparing the results obtained in the previous step with real data. The final step of the application presented in this paper is to compare the derived values in step 9 with the data collected by the shipborne gravimetry data. This is done in order to analyze the results of applying the SMLS and SIMLS methods to the problem under consideration. The minimum, maximum, mean, and standard deviation of the difference between two sets of data-approximated or interpolated by SMLS or SIMLS, respectively, and the BGI shipborne gravimetry data-are computed and analyzed.

\subsection{Deriving gravity acceleration values using SMLS approximation}

In this section, the SMLS method of approximation is used to derive the gravity acceleration values at sea surface in the Persian Gulf. The role of the degree of the harmonic basis functions, the scaling parameter $(\delta)$, which is a function of the degree of the harmonic basis functions, and the two different weight functions (i.e., Gaussian and SWF) is investigated. In what follows, the simple statistical analyses of the difference between derived values-from SMLS approach-and those from the direct observation by BGI institute shipborne gravimetry are given. The analyses are started from the degree 2 for harmonic expansion of the basis functions in Eqn.(8).

\subsubsection{Analysis of data for the harmonic basis functions up to degree 2}

According to Eqn.(20), in order for the coefficients $(c)$ in Eqn.(8) to be estimable, we must have $\left|N_{e}\right| \geq 9$ in Eqn.(6), where || represents cardinality. Hence, the $\delta$ has to be chosen so as to fulfil this requirement.

What is done in this section can be summarized as the following

Computing the gravity accelerations at sea surface, using steps 1-9 in the previous part $\rightarrow$ Subtracting the observed values (collected by shipborne gravimetry) from the computed values $\rightarrow$ Analyzing the differences for different weight functions (Gaussian mode and SWF), various degree for spherical harmonic basis functions, and different $\delta$ values

The following figures show the statistical difference (in milli Gal unit) between the results of approximation by SMLS and those from direct observation. In these figures the maximum, minimum, mean, and the std of the differences are analyzed for different $\delta$ values, beginning with its least possible value, which represents, in each figure, the method of SIMLS.(Note that this is really important: we are implementing SMLS and SIMLS together, although the SMLS is the dominant method.) The used weight function in Fig. 2-4 is SWF. The weight function in Fig. 5-8 is the Gaussian mode. 


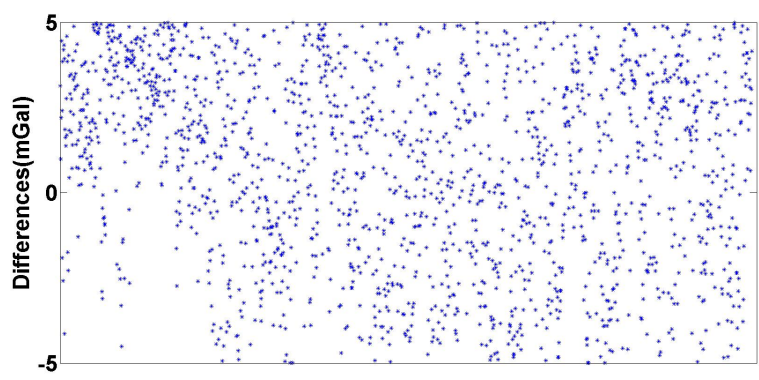

Fig. 2. Scattered diagram of differences between SMLS approximated values and those from shipborne gravimetry

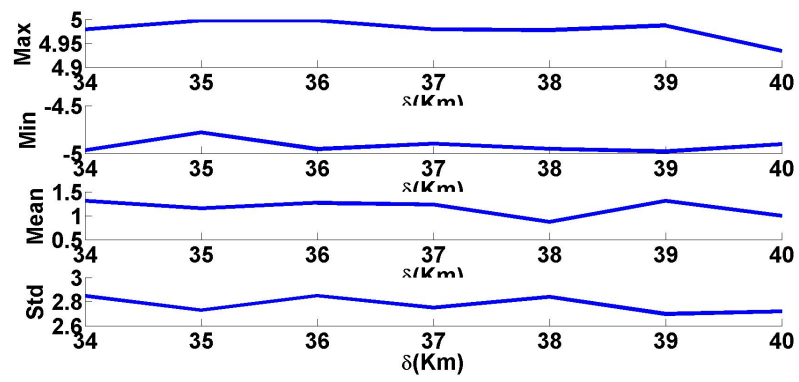

Fig. 3. Statistical properties (maximum, minimum, mean, and std) of the differences between SMLS approximated values and those from shipborne gravimetry, as a function of $\delta$, SWF weight function

Some points about Fig 3 are worth mentioning

- The optimal value for $\delta$ for each of the maximum, minimum, mean, and std criteria is different. No single $\delta$ value can be found such that it causes, simultaneously, the least values of those four criteria.

- Roughly, as $\delta$ increases the difference between derived values and their observed counterparts becomes smaller. The differences are, however, positive, meaning the derived values are greater than observed ones.

- As $\delta$ increases, more points will be used for approximation and the resulting differences are based on more points in the computation steps, changing the configuration of points and as the result, the system of equations to be solved. Thus, the mean and std do not have a regular trend. At $\delta=40 \mathrm{~km}$ the minimum of the degree of freedom of the system of equations in the computation steps (in determining the coefficients $c$ in Eqn.(9)) is 4, i.e. at least 13 points are in the neighborhood of each point. In addition, the least value of std occurs at $\delta=39 \mathrm{~km}$.
- When the mean of the differences increases, most of the points have a lower value than mean and the mode of the maximum is higher.

- The maximum and minimum have, according to the relation in Eqn.(19), a quadratic relation with the normalized distance. This effect is not recognizable in the figures, probably because of the impact of the configuration of neighboring points as $\delta$ increases. In other words, effects of parameters in Eqn.(19) are overshadowed by the effect of neighboring points.

- We can use other criteria for comparing the efficiency of the methods based on $\delta$ values, such as NashSutcliffe Efficiency (NSE) coefficient. In the Fig.4, values of NSE are shown at different $\delta$ values.

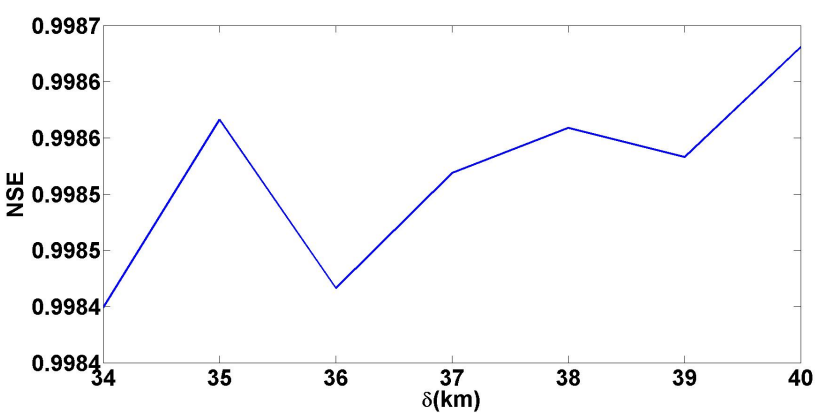

Fig. 4. NSE coefficient, as a function of $\delta$

Thus, one can say based on NSE criterion the best value for $\delta$ is $40 \mathrm{~km}$.

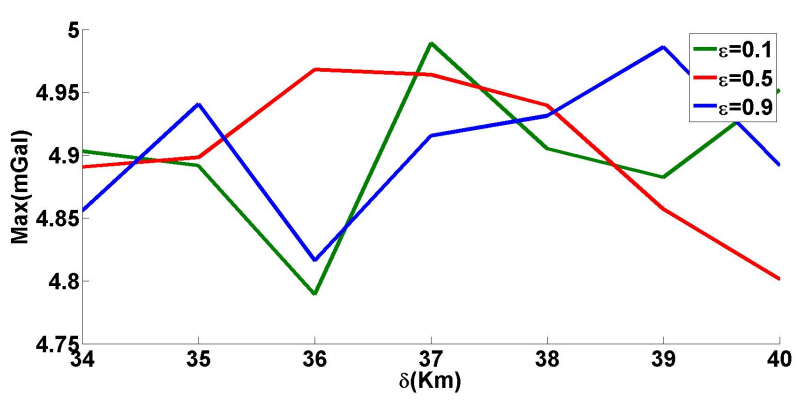

Fig. 5. Maximum of the differences between SMLS approximated values and those from shipborne gravimetry, as a function of $\delta$, Gaussian weight function, $\epsilon=0.1,0.5,0.9$ 


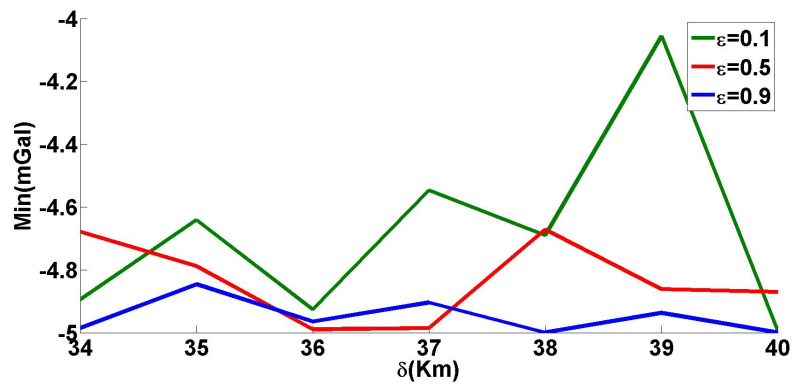

Fig. 6. Minimum of the differences between SMLS approximated values and those from shipborne gravimetry, as a function of $\delta$, Gaussian weight function, $\epsilon=0.1,0.5,0.9$

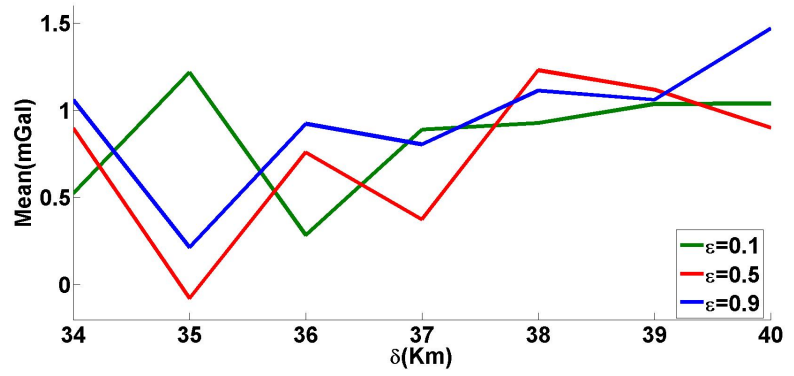

Fig. 7. Mean of the differences between SMLS approximated values and those from shipborne gravimetry, as a function of $\delta$, Gaussian weight function, $\epsilon=0.1,0.5,0.9$

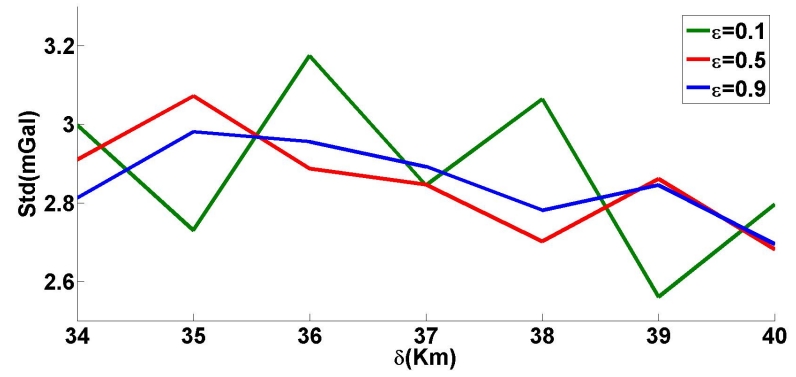

Fig. 8. Std of the differences between SMLS approximated values and those from shipborne gravimetry, as a function of $\delta$, Gaussian weight function, $\epsilon=0.1,0.5,0.9$

The following points about Fig. 5-8 are worth mentioning

- Similar to the SWF weight function, there is no instance for $\delta$ in which all the analysis criteriaminimum, maximum, mean, and std-are optimal.
- With the increase in $\delta$ the maximum of the difference of the results has generally an ascending trend at $\epsilon=$ $0.1,0.9$, but a descending one at $\epsilon=0.5$.

- Compared to the SWF, the Gaussian weight function has a better accuracy in terms of std, when $\epsilon=0.1$, since the minimum of std occurs at $\delta=39 \mathrm{~km}$. However, the changes in std when the Gaussian weight function is used is greater such that in 6 out of $7 \delta$ values the std of this weight function is bigger than that of SWF.

- Although the mean of differences in the Gaussian weight function mode (when $\epsilon=0.1$ ) is, on average, smaller than that of the SWF, the Gaussian weight function mode has an ascending trend. This means that the derived values using SMLS and SIMLS tend to become greater than the shipborne gravimetry values.

- The effect of $\epsilon$ on the minimum of values is first decreasing the absolute value, and then increasing it. This is because the Gaussian weight function has an exponential relation with $\epsilon$, as can be understood from Eqn.(18).

- The effect of $\epsilon$ on the maximum of values is the opposite of its effect on the minimum of differences: first increasing the absolute value, and then decreasing it. Again, this is because the Gaussian weight function has an exponential relation with $\epsilon$.

- With the increase in $\epsilon$ from 0.1 to 0.9 , the mean value of differences has peaks at, respectively, $\delta=$ $35,38,40 \mathrm{~km}$, with generally increasing variability. In addition, it is mostly positive (except when $\delta=$ $35 \mathrm{~km}, \epsilon=0.5$ ), which implies that in general the computed values are greater than the observed values.

- As the parameter $\epsilon$ changes from 0.1 to 0.9 , the value of the std first decreases, then increases, on average. However, at $\epsilon=0.9$ according to Eqn.(30) the values of $d g_{G}$ have a quadratic-exponential behavior, with greater quadratic contribution. As the result, the changes in std values based on $\delta$ is approximately negative-quadratic.

- Unlike the SWF weight function, with the increase in $\delta$ the computed values of more points are greater than the observed values.

- Compared to Gaussian weight function mode, the minimum of the difference of two sets of values changes more uniformly with $\delta$ for SWF. This is because the SWF is a fourth-degree polynomial, smoother than the Gaussian counterpart.

- Unlike the changes in minimum with $\delta$, the changes in maximum of differences in the case of Gaussian weight function are smoother than (or at least as smooth as) SWF. 
- The mean value of differences is, on average, smaller when the weight function is SWF. The changes are, however, of approximately the same magnitude, and in similar directions (both the Gaussian mode and SWF mode first decrease then increase with $\delta$.)

- The Gaussian weight function mode has a smoother changing behavior in terms of std, compared to SWF mode.

\subsubsection{Analysis of data for the harmonic basis functions up to degree 3}

The number of spherical harmonic basis functions in this step is equal to 16, according to Eqn.(20). Thus, the parameter $\delta$ must be chosen so that the 16 coefficients in Eqn.(8) could be estimated. In what follows, we have only analyzed the effect of the scaling parameter on the std of the differences between two different approaches. This is because similar analyses to the ones in Fig. 4-8 can be simply done for the up-to-third order harmonic basis functions. In addition, in the case of our analysis std is a more important criterion than minimum, maximum, and mean: it is a simple yet good means to analyze the accuracy of SMLS approximation method.

Both the SWF and Gaussian weight functions are used in the computations to arrive at Fig. 9. Note that in the Gaussian mode, the parameter $\epsilon$ is chosen to be 0.1, since it has shown better accuracy based on the representations in Fig. 5-8.

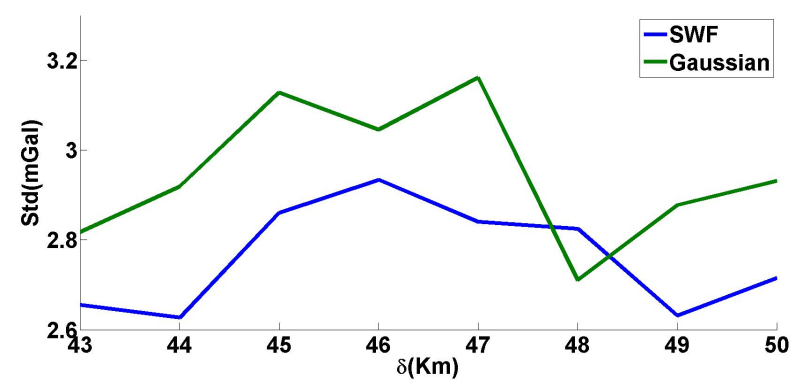

Fig. 9. Std of the differences between SMLS approximated values and those from shipborne gravimetry, as a function of $\delta$, SWF and Gaussian weight functions, $\epsilon=0.1,16$ harmonic basis functions

Note that

- In the SWF mode, the std of differences changes more smoothly with $\delta$ than does Gaussian mode, in addition to being, on average, smaller. This means as the degree of spherical harmonic basis functions increases, the SWF mode works better for the problem that is being analyzed.

- In both cases, SIMLS interpolant works better than SMLS approximant.

- Although it is expected that one can observe the smoothing effect in the SWF mode for results of differences, it is not discernible: as the degree of harmonic basis functions increases, more points have to be found for interpolation and approximation, but the differences first have a higher std value, then a lower one. Note also that one should take into account that the smoothed solution is not always the best one, since it may be far from the state of real data.

- In equivalent $\epsilon$ values $(\epsilon=0.1)$ in Gaussian weight function mode, the impact of increase in the number of harmonic basis functions is, unlike the SWF, "negative", meaning it increases the std, on an average basis.

\subsection{Deriving gravity acceleration using SIMLS interpolation}

\subsubsection{Analysis of data for the harmonic basis functions up to degree 4}

In this section the method of SIMLS is used to interpolate gravity data. It is important to notice that SIMLS was used in the previous section, although the emphasis was on the SMLS method. In fact, the minimum of $\delta$ in Fig 3-8 is chosen so as to have 9 or 16 in the neighborhood of each interpolation points. In this section, however, $\delta=70 \mathrm{~km}$ is fixed and for both the SWF and Gaussian weight functions, the minimum, maximum, mean, and std of the differences between the results of computation by SIMLS and those of observation are analyzed. Hence, we have the following table.

Table 1. Statistical analysis of the differences between SIMLS interpolated values and those from shipborne gravimetry, in mGal unit, 25 harmonic basis functions

\begin{tabular}{c|c|c|c|c}
\hline Weight function & Min & Max & Mean & Std \\
\hline SWF & -4.9965 & 4.9995 & $\mathbf{0 . 8 0 7 0}$ & $\mathbf{2 . 7 6 1 1}$ \\
\hline Gaussian $(\epsilon=0.1)$ & -4.9942 & 4.9956 & $\mathbf{0 . 7 9 2 9}$ & $\mathbf{2 . 9 0 2 8}$ \\
\hline Gaussian $(\boldsymbol{\epsilon}=\mathbf{0 . 5 )}$ & -4.9754 & 4.9993 & $\mathbf{0 . 7 6 9 5}$ & $\mathbf{2 . 8 9 4 8}$ \\
\hline Gaussian $(\boldsymbol{\epsilon}=0.9)$ & -4.9620 & 4.9982 & $\mathbf{0 . 7 8 9 8}$ & 2.9044 \\
\hline
\end{tabular}

Some important points about Table 1 are 
- In terms of mean, the Gaussian weight function mode is better than SWF. However, SWF has a lower std value than does Gaussian weight function.

- As $\epsilon$ increases from 0.1 to 0.9 , the absolute value of minimum gets smaller, maximum first bigger then smaller, mean like the maximum first bigger then smaller, and std first smaller then bigger. This behavior can be explained at least partially by analyzing the quadratic-exponential behavior of the Gaussian weight function derivatives in Eqn.(31).

- Comparing Fig. 9 with Table 1, one can observe that the harmonic basis functions up to degree 3 present a better accuracy, probably because of the stability of the corresponding matrices to be inverted in the computation phase.

- Like the SWF, the $3^{\text {rd }}$ degree harmonic basis functions works better than the $4^{\text {th }}$ expansion in the problem under consideration, comparing Table 1 with Fig. 9.

\subsection{Discussion: comparison of results with the spherical splines method}

Now, one can compare the obtained results with the results in a similar paper on this problem. In (Safari, et al, 2014), controlling the validity of the observed data, collected by shipborne gravimetry approach, is the primary purpose. To this end, method of spherical splines is used in the computational steps 1-10. Here, we have borrowed from (Safari, et al, 2014) the final results of the comparison between interpolation by spherical splines and observed values of gravity acceleration values

Table 2. Statistical analysis of the differences between interpolated values and those from shipborne gravimetry, spherical splines mathematical modelling for residual potential in Eqn.(28). The table is borrowed from (Safari, et al, 2014).

\begin{tabular}{c|c|c|c}
\hline Min(mGal) & Max(mGal) & Mean(mGal) & Std(mGal) \\
\hline-3.49 & 5.99 & 1.37 & 2.61 \\
\hline
\end{tabular}

Considering the Table 1 and Fig. 3-8, one can simply find at least one instance for SMLS and SIMLS such that these two methods work better than spherical splines (except for the minimum, which is always greater (in absolute value) than the result of spherical splines.)

For the maximum criterion the Fig. 3 and Fig. 5, and the Table 1 show that the SMLS and SIMLS methods are better than spherical splines.
For the mean criterion the Fig. 3 and Fig. 6 (except when $\delta=40 \mathrm{~km}$ in Fig. 6), and the Table 1 demonstrate that the SMLS and SIMLS work better than spherical splines.

Finally, if the std is considered for the comparison between the SMLS and SIMLS and spherical splines, one can simply observe that SMLS approximation with Gaussian weight function, $\epsilon=0.1$, up to $2^{\text {nd }}$ degree harmonic basis functions, and with $\delta=39 \mathrm{~km}$ has a lower std value than spherical splines method.

It should be reiterated that the SMLS and SIMLS are numerically more stable, have less computational complexity (thus being faster), and have more parameters to control the accuracy than does the spherical splines method.

Hence, overall, it can be asserted that the SMLS approximation and SIMLS interpolation represent two better methods than the spherical splines, as far as the problem of producing gravity acceleration values at sea surface in the Persian Gulf is considered.

\section{Conclusion}

In this paper, an overview of the SMLS approximation method is presented and its application in geodetic problems is introduced. A simple theory is devised to use the SMLS as an interpolant, which is called SIMLS method. The derivatives of SMLS and SIMLS are presented. The SMLS and SIMLS are then used to produce the gravity acceleration values at sea surface in the Persian Gulf. A thorough analysis of the impact of different parameters in the accuracy level of SMLS and SIMLS methods is presented, by comparing the derived results with the actual, observed values by shipborne gravimetry. The roles of the scaling parameter $(\delta)$, weight functions (Gaussian-with different $\epsilon$ values-and SWF), and the order of the spherical harmonic basis functions are investigated. The results of the paper have been compared with the spherical splines method. It is shown that SMLS and SIMLS represent better ways of approximation and interpolation than does spherical spline. There is at least one instance in which the maximum, minimum, and std criteria by SMLS and SIMLS methods are smaller than those of spherical splines. SMLS and SIMLS methods are faster, more stable, and controllable, in that they have many parameters to change. This is because $\delta$ is chosen so that each point has exactly $(q+1)^{2}$ points in its neighborhood. Also, if the values to be interpolated are randomly selected, then the first choice would be the Gaussian weight function. In addition, if smoothness is consid- 
ered, it is better to use SWF. Besides, if high stability is required the lower the degree of harmonic expansion $(q)$ the better, whereas if higher precision is required, it is better to increase the value of $q$. So, the degree of harmonic expansion is a balance between stability and precision.

There are many other applications to the SMLS and SIMLS in Geosciences, including Oceanography. They can be used to approximate and interpolate data in many cases other than gravity field. For instance, TEC (Total Electron Content) can be interpolated using SIMLS and be compared with other methods such as biharmonic spherical splines, presented in (Keller, et al, 2019). The solution of differential equations is another application that can be pursued by researchers. In general, the geodetic community would benefit from having more accurate and computationally faster mathematical models for solving problems, and SMLS and SIMLS are two powerful methods that could be used to achieve this goal.

Acknowledgement: The author is willing to acknowledge the BGI institute staff for providing the shipborne gravimetry dataset used in this study. Also, M. C. Kim, B. D. Tapley, C. K. Shum, and J. C. Ries are gratefully thanked for providing MSL data. The anonymous reviewer, whose invaluable help improved this paper is acknowledged, as well.

\section{List of acronyms:}

SMLS: Spherical Moving Least Squares

SIMLS: Spherical Interpolating Moving Least Squares

\section{References}

Akhtar N. and Michel V., 2012, Reproducing-kernel-based splines for the regularization of the inverse ellipsoidal gravimetric problem, Applicable Analysis, 91, 12, 2105-2132.

Ardalan A. A. and Grafarend E. W., 2001, Ellipsoidal geoidal undulations (ellipsoidal Bruns formula): case studies, J Geod, 75, 544-552.

Baramidze V., Lai M. J. and Shum C K., 2006, Spherical splines for data interpolation and fitting, SIAM, 28, 1, 241-259.

Cheng Z.Q., Wang Y.Z., Li W. B., Xu K., Dang G. and Jin S.Y., 2008, A survey of methods for moving least squares surfaces, IEEE/EG Symposium on Volume and Point-Based Graphics, 9-23.

Dawes R. and Thompson D L., 2007, Interpolating moving leastsquares methods for fitting potential energy surfaces: computing high-density potential energy surface data from lowdensity ab initio data points, J. of Chem. Phys., 126, 18, 18411848.

Rapp R. H., 1998, The development of a degree 360 expansion of the dynamic ocean topography of the POCM-4B global circu- lation model, NASA/CR-1998-206877, Goddard Space Flight Center, Greenbelt, MD.

Freeden W., Nashed M. Z. and Schreiner M., 2018, Spherical sampling, Springer.

Freeden W., Gervens T. and Schreiner M., 1998, constructive approximation on the sphere, Clarendon press, Oxford University Press.

Freeden W. and Schreiner M., 2009, Spherical functions of mathematical Geosciences, Advances in Geophysical and Environmental Mechanics and Mathematics, Springer.

Freeden W., 1981, On spherical spline interpolation and approximation, Math. Meth. in the Appl. Sci., 3, 1, 551-575.

Jekeli C., 1988, The exact transformation between ellipsoidal and spherical harmonic expansions, Manuscripta geodaetica, 13, 1, 106-113.

Kawano A., Tokmakov I. V., Thompson D. L., Wagner A. F. and Minkoff M., 2006, Interpolating moving least-squares methods for fitting potential-energy surfaces: further improvement of efficiency via cutoff strategies, J of Chem. Phys., 124, 5, 541545.

Keller W. and Borkowski A., 2019, Thin plate spline interpolation, J of geodesy, 93, 1, 1251-1269.

Kim M. C., Tapley B. D., Shum C. K., Ries J. C., 1995, Mean sea level model, Center for Space Research, TOPEX/POSEIDON working team meeting, Pasadena, California.

Kiani M., 2020, Local geoid height approximation and interpolation using moving least squares approach, J of geodesy and Geodynamics. https://doi.org/10.1016/j.geog.2019.12.003

Kiani M. and Chegini N., 2019, Ellipsoidal spline functions for gravity data interpolation and smoothing, Journal of EOGE. doi:10.22059/eoge.2020.290542.1065

Kiani M., 2020, Template-based smoothing functions for data smoothing in Geodesy, J of geodesy and Geodynamics. https://doi.org/10.1016/j.geog.2020.03.003

Lancaster P. and Salkauskas K., 1981, Surfaces generated by moving least squares methods, J of Math. Comput, 37, 155, 141-158.

Maisuradze G. G. and Thompson D. L., 2003, Interpolating moving least-squares methods for fitting potential energy surfaces: illustrative approaches and applications, J of Chem. Phys, 107, 37, 7118-7124.

Safari A., Sharifi M. A., Amin H. and Foroughi I., 2014, Gravity acceleration at the sea surface derived from satellite altimetry data using harmonic splines, J of Earth and Space Phys, 40, 3, 35-46.

Wahba G., 1981, Spline interpolation and smoothing on the sphere, SIAM, 2, 1, 5-16.

Wahba G., 1990, Spline models for observational data, SIAM, Philadelphia, Pennsylvania.

Wendland H., 2000, Moving least squares approximation on the sphere, Math. Meth. in CAGD, 517-526. 